\begin{tabular}{ccc}
\hline & International Journal of Engineering \& Technology, $7(2.32)(2018) 377-380$ \\
SPC & International Journal of Engineering \& Technology \\
Website: www.sciencepubco.com/index.php/IJET & Research paper \\
\hline
\end{tabular}

\title{
Cost Estimation of the Models Using Harmony Search
}

\author{
Dr. T. Vijaya Saradhi ${ }^{1}$, A. Lakshmi Pravallika ${ }^{2}$,M. Manoj ${ }^{3}$ \\ ${ }^{1,2,3}$ Dept of CSE , K L E F,Vaddeswaram, India \\ *Corresponding author E-mail: pravallikaalgapuri@gmail.com
}

\begin{abstract}
To estimate the cost of model accurately on which the software is functioning is one of the most important things in the software project. But due to the varying nature of the software, and complexity, accurate cost estimation of software has become difficult. Ascertaining the cost of the software at the beginning stage is helpful for designing the other activities of software development. Former estimation of the needed exertion to Creating programming need benefited the advancement acknowledging those provision about Meta heuristic streamlining calculations. These calculations need aid possibility and might a chance to be connected Likewise functional devices for programming expense estimation. In the recent times Meta- heuristic algorithms with high accuracy have brought a great improvement in the field of the software engineering. In this paper we have discussed about the one of the algorithm which help in software cost estimation which is Harmony Search.
\end{abstract}

Keywords:Meta Heuristic techniques, Cost Estimation, Harmony Search Algorithm.

\section{Introduction:}

Cost estimation of software project is a very significant and important stage of a project development as it affects and contributes to the controlling process. This estimation is not exclusively to pick up the accuracy, but also to manage the expense furthermore and set the limits of the project. The customer wants their software product with high reliability and less cost. Reliability and cost are the two important pillars that estimate the success of software. If the estimation of the software is wrongly done, then it is not only going to affect the product but also the reputation of the company. Value estimation software program is a complex hobby that calls for information of many key attributes about the task for which the estimate is being built. Growing accurate software cost estimates requires knowledge of the subsequent parameters: the sizes of predominant deliverables, stability of the measuring tool and instruction, Line of Code(LOC), effort, time and manuals. There are many intuitive models for the cost estimation. Similarly, reasoning and expert opinion is the best way of the calculation of the cost. Estimation based on similarity means comparing the ongoing software projects intended for estimation with the previous software projects. On the other hand, the expert opinion, matches the previous similar projects with each other and finally does the estimation of the projects. Over the past two- three decades, various models have been used to estimate the Cost in the various software development processes. Exact cost estimation is very important for the project because if we do not conclude it then the cost of the product can increase from $25 \%$ to $75 \%$. In many software projects the cost estimation is done on the base of the lines of code (LOC) and the functional point. In this paper we have discussed about the different models which helps to estimate the cost models.

\section{Related works}

In past, many works had been deduced on the software cost estimation. Accurate cost estimation is highly important to finish the project on time. It helps in the prior estimation of costs because of which unnecessary expenditure in the project can be avoided. This helps the developers to pay more attention on the reliability and quality of the project. Artificial Intelligence is one of best way for testing and the evaluations of the software at any stage. These sections contain some of the models which evaluate the software cost.

Hybrid PSO-FCM and PSO-LA models have been proposed for the SCE (Software Cost Estimation). Particle swarm optimization (PSO) [3] is a computational technique that minimises the issue by means of trying repetitively to enhance a candidate solution with respect to a given measure of first-class. It figure out a hassle with the aid of having a populace of candidate answers and moving those particles around in the search-space according to easy mathematical formulae over the particle's place and speed. Every step of the particle is motivated by way of its local pleasant recognized role, but is also manage towards the first-rate-recognized point inside the search-area, which might be up to date as higher positions are determined through different particles. This is expected to move the swarm closer to the first-rate solutions. This model calculates the minimum inter and the intra cluster distance to perform the fitness test and the software efficiency. FCM gather the best cluster and then perform the fitness. Learning Automata (LA) is performed to improve the PSO algorithm by adjusting the behavior of the particles. This algorithm also helps in understanding the parameters which affects the cost of the software.

COCOMO: [2] COCOMO is the abbreviation of Constructive Cost Model. This is an algorithm strategy for software price 
estimation this is evolved by Barry W. Boehm in 1981 to offer estimation of wide variety man-months it will take to expand a software product. COCOMO predicts the efforts and time of software program product based totally on size of software program. COCOMO encompass little by little technique for estimation of software fee estimation(SCE). It is an skilled version done by means of amassing the records from many software initiatives. The intention of this estimate is to minimise the suggest mean magnitude relative error which whilst applied can result in $21 \%$ optimization. essentially, there are two types of COCOMO they are COCOMO 1 and COCOMO 2. COCOMO 1 is the primary degree effort calculation approach whereas $\mathrm{CO}$ COMO 2 is superior stage. This version uses regression method to infer the cost of software. COCOMO is a hierarchy model which incorporates:

\section{Basic COCOMO \\ 2. Intermediate COCOMO \\ 3. Detailed COCOMO}

Basic COCOMO: - It estimates the cost of the software based on the size of the software. It is applied to the following projects: -

1. Organic projects
$2 . \quad$ Semi-detached projects
$3 . \quad$ Embedded projects
\begin{tabular}{|l|l|}
\hline Basic COCOMO & $\mathrm{E}=\mathrm{a}(\text { Size })^{\mathrm{b}}$ \\
\hline Organic & $\mathrm{E}_{\mathrm{B}-\mathrm{O}=2.4^{*}(\mathrm{~L})}^{1.05}$ \\
\hline Semi-detached & $\mathrm{E}_{\mathrm{B}-\mathrm{S}=3.0^{*}(\mathrm{~L})}^{1.12}$ \\
\hline Embedded & $\mathrm{EB}-\mathrm{E}=3.6^{*}(\mathrm{~L})^{1.20}$ \\
\hline
\end{tabular}

Intermediate COCOMO: - It also estimates the cost of software on the bases of the-lines of code (LOC) but it also includes a set of cost factors attributes.

The intermediate is based upon the given formula:-

$$
\mathrm{E}=a_{i}(\mathrm{KLOC}){ }^{\left(\mathrm{b}^{\prime}\right)}(\mathrm{EAF})
$$

Where $a_{i}$ denotes the coefficient and $b_{i}$ the exponent. EAF stand for the Effort Adjustment Factor.

Detailed COCOMO: - the software is sub divided into modules and each module is passed through the COCOMO model to estimate the effort and sum of the effort. The phase of the detailed COCOMO is: -

$\begin{array}{ll}\text { 1. } & \text { Planning and requirement } \\ \text { 2. } & \text { System design } \\ \text { 3. } & \text { Detailed design } \\ \text { 4. } & \text { Module cost and test } \\ \text { 5. } & \text { Cost constructive model } \\ \text { 6. } & \text { Integration and test }\end{array}$

Functional Points have been described in 1979 in Measuring Application Development productivity by of Allan Albrecht at IBM. It's miles the maximum easy and easier way of calculating the cost of software program. Function point analysis is particularly used to estimate the size of the undertaking or application. To estimate the attempt of any application length is a completely crucial factor In [5] direct technique size is predicted as lines of Code(LOC). And in indirect method the function points are used to estimate the size. FPA to start with decomposes a venture or application into its data fragments and assignable capabilities. The records features suggest the capability provided to the person with the aid of attendant to their inner and outside needs related to the statis- tics, while the assignable capabilities indicate the capability furnished to the person related to the processing.

$$
\mathbf{H M}=\left[\begin{array}{ccc|c}
x_{1}^{1} & \cdots & x_{n}^{1} & f\left(\mathbf{x}^{1}\right) \\
\vdots & \ddots & \vdots & \vdots \\
x_{1}^{h m s} & \cdots & x_{n}^{h m s} & f\left(\mathbf{x}^{h m s}\right)
\end{array}\right] .
$$

The FP is based upon the five factors: -

1. Internal logic files (ILF)

2. External interface files(EIF)

3. External inputs(EI)

4. External outputs(EO)

5. External inquiries(EI)

As soon as the function is identified and categorized into a type, it is then assessed for complexity and assigned many characteristic factors. The categorized features imply the relative complexity as low, medium and excessive. The information functions relative useful complexity depends on the variety of facts detail kinds (DETs) and the range of record element kinds (RETs). The assignable capabilities are restricted based totally at the range of file types referenced (FTRs) and the quantity of DETs. The ILF and EIF are categorized as data functions and the EI, EO, EI(external inquiries) are classified as transactional functions. Each of them maps to an end-user business characteristic, inclusive of a records access for an input or a person question for an Inquiry. These five factors determine help to figure out the complexity metrics of the software which is known as the functional complexity.

Harmony search Algorithm: Harmony search is a phenomenon "mimicking" meta- heuristic algorithm, used in looking of an optimized cost estimation procedure. This set of rules turned into first offered by Zong Woo Geem in 2001. Harmony search algorithm is stimulated by way of the herbal system of playing song and attempts to find an exceptional harmony inside the process. Here, a thrilling together with quality harmony (a whole nation of concord) is looked for and also in the same way to achieve the criteria of harmony magnificence. The set of rules uses stochastic or random values. The quest follows the manner era of answer vectors, and movements during trouble fixing from hassle area to the most effective area within the specified constraints. The harmonic search specially proceeds based on probabilistic approach. Within the homogeneous manner, look for the choicest solution in engineering and optimization problems is meant to take vicinity based totally on the goal characteristic.

The Harmony search set of rules proceeds inside the following steps:

To create a new vector of variables (the harmony vector) in HS set of rules, each variable's fee is decided in line with one of the following regulations [7]:

1. select one available value from $\mathrm{HM}$.

2. select the cost next to the value that is chosen from HM

3 . select the value for the variable from their felony constraint stochastically.

Stage 1- Defining the optimization trouble and initializing set of rules parameters: The optimization problem mainly includes of maximizing or minimizing the goal feature $\mathrm{F}(\mathrm{x})$; in the constraints. Parameters initialized here are: the variety of selection variables (M) and it's alternate c programming language, concord memory length (HMS), the opportunity of choosing a variable from harmony reminiscence (HMCR), the opportunity of modifying the variable selected from concord reminiscence (PAR), modification bandwidth (BW) and termination criteria (biggest frequency) [7]. 
Stage 2- Initializing harmony memory: The memory initialization is completely stochastic, and the number of generated vectors matches the size of harmony reminiscence. The primary concord reminiscence is stored with the values corresponding to the objective characteristic. Following parent indicates the structure of concord reminiscence [7]. each line represents a harmony of $\mathrm{N}$ variables and HMS represents the size of the harmony memory.

Harmony memory structure

Stage 3- generation of a brand-new harmony from reminiscence: the new harmony vector is generated in step with the above said rules.

Stage 4- Updating concord memory: If the brand-new harmony is better and ideal than the worst present concord, the present one is replaced with the modern-day one with the corresponding objective function value and the worst harmony of the memory is eliminated

Stage 5- Monotony of stage three and four till termination condition is met: The windup condition is commonly stated as satisfaction of one situation or accomplishing the very best frequency.

\section{Proposed Theory}

Based on all the observations from various cost estimation models, we can predict the best model for cost optimization and estimation. Various models have various drawbacks. In the PSO-FCM model, the models do not have any type of overlapping calculation. It works best for the AI software. But the main drawback of the model is that it is sensitive to the noise. And it also has weak local search ability. This hinders the optimization of the solutions. The next model studied is the COCOMO model. It is a formal estimation method. This comes under the parametric model. The parameters play an important role and is easy to interpret. It looks over many factors for the estimation. But the main drawback is that it ignores the hardware issues. The hardware and software compatibility is highly important to develop any software. If the selected hardware do not support the current software version them the cost of the software can increase because of the encountered errors. Another model is the Functional point analysis. It is also a formal estimation method, and this comes under the size based estimation. FP measure the size of the solution rather than the size of the problem. This may sometimes cause difficulty in cost estimation because, without knowing the problem size, it is difficult to interpret the size of the solution. Hence to avoid these drawbacks, harmony search algorithm is preferred to estimate the cost of a software rather than the traditional methods. As the harmony search is conventional metaheuristic algorithm, it gives a sufficiently excellent method to an optimization problem, especially with incomplete or imperfect information or restricted computation capability. Metaheuristics sample a set of solutions which is too massive to be absolutely sampled. Metaheuristics can also make few assumptions about the optimization problem being solved, and so they may be usable for an expansion of issues, and as it's far a standard set of rules it has an upper hand over the conventional algorithms. Another advantage is that as it uses the dynamic memory, the worst solutions are replaced with the current optimal solutions, and finally we are left with a set of optimal solutions. One more advantage is that as it is a generic algorithm, it can deal with the complexity and the parallelism as well. All these advantages of harmony search make it a optimal and preferred cost estimation technique for software's. Hence, we propose the use of harmony search algorithm as a better cost estimation technique rather than the other studied models because of its generic and dynamic nature.

\section{Conclusion and Future Scope}

Since the PSO-FCM model are sensitive to the noise and have weak local search where Harmony search algorithm does not have such problem. Therefore, HAS is better than the PSO-FCM. On the other hand, the FP model and the COCOMO is one of the formal estimation methods but totally based on the size of the code and the factors. It does not work when complexity is very high, and the size of the solution is not present. The HSA does not depend upon the size of the software or the solution and it is designed in such a way that the complexity calculation is easier for it Thus, HSA is the best model for the cost estimation. Harmony Search algorithm hybridized with other algorithms such as particle swarm or ant colony optimization can give more optimized results than harmony search alone. The future work can be performed in hybridizing the harmony search with other varieties of optimization algorithms to estimate the software cost, reliability and obtain the optimal results.

\section{References}

[1] Aashima Kundu, Vikas Sethi "Parameter Estimation of COCOMO II using Simulated Annealing" 2012.

[2] A Research Paper on Software Cost Estimation COCOMO Model: A Survey, Priyanka Jain , Aditi

[3] Jain, GADL Journal of Inventions in Computer Science and Communication Technology (JICSCT) ISSN(O): 2455-5738 Volume 3 - Issue 2, March-April, 2017.

[4] Kennedy, J.; Eberhart, R. (1995). "Particle Swarm Optimization". Proceedings of IEEE International Conference on Neural Networks. IV. pp. $1942-1948$

[5] Rohit Kumar Sachan, Ayush Nigam, Avinash Singh, Sharad Singh, Manjeet Choudhary, Avinash Tiwari and Dharmender Singh Kushwaha "Optimizing Basic COCOMO Model using Simplified Genetic Algorithm" Twelfth International Multi-Conference on Information Processing-2016 (IMCIP-2016).

[6] Software Cost Estimation using Function Point with NonAlgorithmic Approach By Dr. N. Balaji, N. Shivakumar \& V. Vignaraj Ananth, Global Journal of Computer Science and Technology Software \& Data Engineering Volume 13 Issue 8 Version 1.0 Year 2013

[7] A.F. Sheta, "Estimation of the COCOMO Model Parameters Using Genetic Algorithms for NASA Software Projects", Journal of Computer Science, Vol. 2, No. 2, pp.118-123, 2006.

[8] A. Rastegar, "Development harmony search method for solving optimization problems: A Case Study in production scheduling parallel machines", Journal of Industrial Engineering studies in production systems ,pp. 57-71,2013.

[9] Ziauddin, Sh. K.Tipu, Kh. Zaman, Sh. Zia, "Software Cost Estimation Using Soft Computing Techniques", Advances in Information Technology and Management (AITM), Vol. 2, No. 1, pp. 233-238, 2012.

[10] Prasad Reddy P.V.G.D, Hari CH.V.M.K, S. Rao.T, "Multi Objective Particle Swarm Optimization for Software Cost Estimation", International Journal of Computer Applications, Vol. 32, No.3, pp. 13-17, October 2011

[11] V. Kh. Bardsiri, "An Optimization-Based Method to Increase the Accuracy of Software Development Effort Estimation", Journal of Basic and Applied Scientific Research , pp. 159-166,2013.

[12] A. O. Gazi5ina, "The Optimization of COCOMO Model Coefficients Using Genetic Algorithms", Information Technology and Management Science ,pp.45-51,2012

[13] M. A. Mahdavi, “ An improved harmony search algorithm for solving optimization problems", App1 Math Comput ,pp.15671579,2007

[14] V. Khatibi, and D. Jawawi, " Software Cost Estimation Methods: A Review", Journal of Emerging Trends in Computing and Information Sciences ,vol 2 ,pp. 21-29,2011

[15] S.G. MacDonell, A.R. Gray, "A Comparison of Modeling Techniques for Software Development Effort Prediction", in Proceedings of International Conference on Neural Information Processing and Intelligent Information Systems, pp. 869872, 1997.

[16] Z.A. KHALIFELU, F.S. GHAREHCHOPOGH, "A New Approach in Software Cost Estimation Using Regression Based Classifier", 
AWER Procedia Information Technology \& Computer Science Journal, Vol. 2, pp. 252-256, December 2012.

[17] F. S. Gharehchopogh, "A Novel Particle Swarm Optimization Approach for Software Effort Estimation", International Journal of Academic Research , vol.6, PP.69-76,2014.

[18] Barry W. Boehm, Ricardo Valerdi "Achievements and Challenges in Cocomo based Software Resource Estimation" IEEE software (Volume: 25, Issue:5, Sept.-Oct. 2008) 